\author{
VIRAL HEPATITIS
}

\title{
TM6SF2 rs58542926 is not associated with steatosis and fibrosis in large cohort of patients with genotype 1 chronic hepatitis $C$
}

\author{
Salvatore Petta ${ }^{1}$, Marcello Maida ${ }^{1}$, Stefania Grimaudo ${ }^{1}$, Rosaria M. Pipitone ${ }^{1}$, Fabio S. Macaluso ${ }^{1}$, Daniela Cabibi ${ }^{2}$, \\ Calogero Cammà ${ }^{1}$, Vito Di Marco ${ }^{1}$, Sandro Sferrazza ${ }^{1}$ and Antonio Craxi ${ }^{1}$ \\ 1 Section of Gastroenterology, Di.Bi.M.I.S., University of Palermo, Palermo, Italy \\ 2 Cattedra di Anatomia Patologica, University of Palermo, Palermo, Italy
}

Liver Int. 2016; 36: 198-204. DOI: 10.1111/liv.12918

\begin{abstract}
Background \& Aims: We tested the putative association of the rs58542926 variant of TM6SF2, a recently described genetic determinant of nonalcoholic fatty liver disease, with steatosis and fibrosis in genotype 1(G1) chronic hepatitis $\mathrm{C}(\mathrm{CHC})$ patients. Methods: A total of 694 consecutively biopsied Caucasian G1 CHC patients were genotyped for TM6SF2 rs58542926, IL28B rs 12979860 and PNPLA3 rs738409. Steatosis was classified as absent (<5\%), mild-moderate(5-29\%) and severe $(\geq 30 \%)$, Fibrosis was considered severe if $=F 3-F 4$. Results: Carriers of TM6SF2 rs58542926 (6.3\% of patients) exhibited lower serum levels of cholesterol $(P=0.04)$ and triglycerides $(P=0.01)$, but a similar distribution of steatosis severity $(P=0.63)$, compared to noncarriers. Prevalence and severity of steatosis were reduced in IL28B C allele carriers $(P=0.005)$ and elevated in PNPLA3 $\mathrm{G}$ allele carriers $(P<0.001)$. After adjustment for age, gender, body mass index and homoeostasis model assessment score, steatosis severity was independently associated with IL28B rs12979860 (odds ratio $[O R]$ 0.69, 95\% confidence interval $[\mathrm{CI}] 0.55-0.86, P=0.001$ ) and PNPLA3 rs738409 (OR 1.84, 95\% Cl 1.46-2.83, $P<0.001$ ), but not TM6SF2 rs58542926 (OR 1.48, 95\% Cl 0.82-2.69, $P=0.19)$. Variants of TM6SF2 (30.9\% vs. 25\%, $P=0.40), I L 28 B$ and PNPLA3 were not directly associated with fibrosis severity, although variants of IL28B and PNPLA3 promoted steatosis (OR 1.36, 95\% Cl 1.06-1.75, $P=0.01$ ) that in turn is associated with severe fibrosis. Conclusions: In G1 CHC patients, TM6SF2 rs58542926 does not affect the histological severity of liver damage. However, IL28B rs12979860 and PNPLA3 rs738409 modify steatosis.
\end{abstract}

\section{Keywords}

CHC - IL28B - PNPLA3 - steatosis - TM6SF2

The estimated global prevalence of hepatitis $\mathrm{C}$ virus (HCV) infection is $2.2 \%$, corresponding to about 130 million HCV-positive persons worldwide, most of whom are chronically infected (1). HCV is one of the main causes of cirrhosis, hepatocellular carcinoma and liver transplantation in Western countries. The recent development of interferon-free therapeutic regimens to treat $\mathrm{HCV}$ would allow for the eradication of infection in more than $90 \%$ of treated patients and a strong improvement in the natural history of their liver disease
(2). However, the impossibility of treating all infected patients (because of cost) and the presence of numerous cofactors that accelerate the progression and complications of $\mathrm{HCV}$-associated liver disease have prompted clinical research to understand the modifiers of liver damage.

In this complex landscape, hepatic steatosis and some of its genetic determinants exert key roles as modifiers of liver disease severity in patients with genotype 1 (G1) chronic hepatitis C (CHC). Specifically, steatosis has

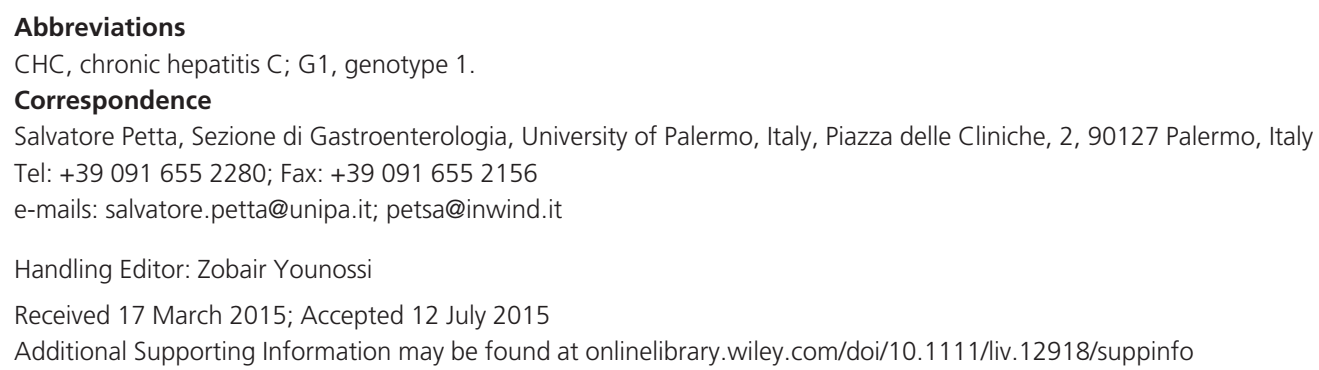




\section{Key points}

- The TM6SF2 rs58542926 gene variant has been associated with the presence and histological severity of nonalcoholic fatty liver disease.

- Previously reported preliminary data in hepatitis C virus-infected patients suggested that TM6SF2 rs58542926 modulates steatosis.

- In this study of the largest cohort of genotype 1 chronic hepatitis $\mathrm{C}$ patients, we found that the TM6SF2 rs58542926 gene variant is not linked to the presence or severity of fatty liver or fibrosis.

- We confirmed the association of variants of PNPLA3 and IL28B in modifying steatosis.

been associated with an increased severity of liver fibrosis (3). It also has emerged as a predictor of fibrosis progression (4) and hepatocellular carcinoma occurrence (5). Variants of two genes, PNPLA3 and IL28B, have been reported to affect the prevalence and severity of steatosis, the severity of fibrosis and the prognosis of liver disease in patients with CHC (6-16). Specifically, the PNPLA3 rs738409 and IL28B rs12979860 variants are the strongest known genetic risk factors for occurrence of nonalcoholic fatty liver disease (NAFLD) (6) and for response to interferon/ribavirin therapy in $\mathrm{CHC}$ (7) respectively.

A recent exome-wide study identified the rs58542926 variant of TM6SF2, a gene that modulates lipid export from the liver, as a new genetic determinant of NAFLD (17). This variant is a nonsynonymous $\mathrm{C}>\mathrm{T}$ polymorphism. Recent studies by others and us have demonstrated that this variant increases the risk that NAFLD patients will develop liver fibrosis and steatohepatitis (18-20). Only two studies have examined the effects of the TMS6SF2 variant in CHC patients $(21,22)$. However, the inclusion of $\mathrm{CHC}$ patients with different $\mathrm{HCV}$ genotypes complicates the interpretation of these studies. Therefore, a large study of this variant in $\mathrm{CHC}$ patients with a single HCV genotype has been necessary. The aim of this study was to assess the putative association between the TM6SF2 polymorphism and histological steatosis, as well as the impact on the severity of liver fibrosis in a large cohort of G1 CHC patients.

\section{Patients and methods}

This study cohort comprised $694 \mathrm{CHC}$ patients who were consecutively enrolled at the Gastrointestinal and Liver Unit of the University Hospital Palermo, Italy. The following inclusion criteria were applied: (a) diagnosis of G1 CHC based on HCV serology and viral RNA, (b) histological diagnosis of CHC by liver biopsy, (c) self-reported alcohol consumption of less than $20 \mathrm{~g} /$ day in the last 12 months, as evaluated by a questionnaire and (d) availability of DNA for genotyping.
Patients were excluded from this study if they were co-infected with either the hepatitis B virus or HIV.

This study was carried out in accordance with the principles of the Helsinki Declaration and its appendices, as well as with local and national laws. Approval was obtained from the AOUP Paolo Giaccone of Palermo's Internal Review Board and its Ethics Committee. Written informed consent was obtained from all patients.

\section{Clinical and laboratory evaluation}

Clinical and anthropometric data were collected at the time of liver biopsy. Patients with a body mass index (BMI) of $30 \mathrm{~kg} / \mathrm{m}^{2}$ or greater were classified as obese. Impaired glucose tolerance and type 2 diabetes mellitus (T2DM) were diagnosed on the basis of the revised criteria of the American Diabetes Association (fasting blood glucose of 100-125.9 mg/dl for impaired glucose tolerance or $\geq 126 \mathrm{mg} / \mathrm{dl}$ for T2DM) (23). In patients with a previous diagnosis of T2DM, current therapy with insulin or oral hypoglycaemic agents was recorded.

A 12-h overnight fasting blood sample was drawn at the time of biopsy to determine the serum levels of ALT, total cholesterol (TC), triglycerides (TG), plasma glucose, insulin and platelet count. Insulin resistance (IR) was determined according to the homoeostasis model assessment (HOMA) method (24): Insulin resistance $($ HOMA-IR $)=$ Fasting insulin $(\mu \mathrm{U} / \mathrm{ml}) \times$ Fasting blood glucose $(\mathrm{mmol} / \mathrm{L}) / 22.5$.

\section{Genotyping}

DNA was extracted from peripheral blood collected at the time of enrolment in all patients. Genotyping for TM6SF2 (rs58542926), PNPLA3 (rs738409) and IL28B (rs12979860) was carried out by using the TaqMan SNP genotyping allelic discrimination method (Applied Biosystems, Foster City, CA, USA). Genotyping calls were made by using the SDS software package (v.1.3.0, ABI Prism 7500, Foster City, CA, USA). Genotyping was conducted in a blinded fashion relative to patient characteristics.

\section{Histopathology}

A liver biopsy was performed for all patients. Liver histology was used to determine the extents of steatosis, hepatic necroinflammation and fibrosis. Steatosis was graded as absent $(<5 \%)$, mild-moderate (5-29\%) or severe $(\geq 30 \%)$. Liver fibrosis and necroinflammatory activity were classified according to the Scheuer score (25).

\section{Statistical analysis}

Our study had a power of more than $80 \%$ for detecting an odds ratio (OR) of 1.5 for steatosis for the TM6SF2 
rs58542926 variant, with a significance of 5\%. Continuous variables were summarized as the mean $\pm S D$, and categorical variables as the frequency and percentage. The $t$ - and $\chi^{2}$ tests were used where appropriate. Multiple ordinal regressions were used to assess variables associated with the severity of steatosis. Multiple logistic regression models were used to assess the factors associated with severe fibrosis. ORs and 95\% confidence intervals (CIs) are reported.

As candidate risk factors, we used gender, age, BMI, HOMA score, TM6SF2 rs58542926 genotype, PNPLA3 rs738409 genotype and IL28B rs12979860 genotype. In the analysis, the TM6SF2 E172K variant was coded in a dominant genetic model because of its relatively low allele frequency $(19,20)$. The PNPLA3 and IL28B variants were coded in an additive model. Analyses were performed by using the sPSs software package.

\section{Results}

\section{Patient features and histology}

Baseline characteristics of the 694 patients are shown in Table S1. The mean age was 53 years, and $54 \%$ of patients were male. Obesity and T2DM each were observed in about $13 \%$ of patients. Mean TC and TG levels were within the normal range. The mean HOMA value was 3.37. One-third of patients had severe fibrosis. Steatosis was observed in about half of cases, and 17\% had high-grade steatosis.

Distributions of the IL28B rs12979860 and PNPLA3 rs738409 genotypes are reported in Table S1. For the TM6SF2 rs58542926 variant, $93.7 \%$ of patients were homozygous for the $\mathrm{C}$ allele, and $6.3 \%$ of cases carried the $\mathrm{T}$ variant. Only one patient was homozygous for the $\mathrm{T}$ allele. Genetic frequencies were in Hardy-Weinberg equilibrium. In our cohort, the metabolic profile differed according to the TM6SF2 genotype (Table 1). Patients carrying the rs58542926 variant were characterized by lower serum levels of TC $(P=0.04)$ and TG $(P=0.01)$ compared to noncarriers.

\section{Associations of gene variants with steatosis severity in G1 CHC patients}

Patients carrying the TM6SF2 rs58542926 variant had a similar distribution of steatosis severity as noncarriers. Percentages of patients with absent, mild-moderate and severe steatosis were $54.8 \%, 28.8 \%$ and $16.4 \%$, respectively, in carriers compared to $47.7 \%, 31.8 \%$ and $20.5 \%$, respectively, in noncarriers $(P=0.63$, Fig. 1A). Prevalence and severity of steatosis were lower in patients carrying the $I L 28 B$ C allele $(P=0.005$, Fig. $1 \mathrm{~B})$ and elevated in those carrying the PNPLA3 G allele $(P<0.001$, Fig. $1 \mathrm{C})$ compared to the corresponding noncarriers.

A multivariate ordinal regression analysis adjusted for age, gender, BMI and HOMA score showed that
Table 1. Characteristics of 694 patients with genotype 1 chronic hepatitis C, according to TM6FS2 rs58542926 genotype

\begin{tabular}{|c|c|c|c|}
\hline Variable & $\begin{array}{l}\text { TM6SF2 } \\
\text { CC } \\
n=650(93.6)\end{array}$ & $\begin{array}{l}\text { TM6SF2 } \\
\text { TC/TT } \\
n=44(6.4)\end{array}$ & $\begin{array}{l}P \\
\text { value }\end{array}$ \\
\hline Age (yrs) & $53.7 \pm 11.9$ & $52.9 \pm 11.7$ & 0.65 \\
\hline Male gender (\%) & 53.8 & 61.3 & 0.33 \\
\hline $\begin{array}{l}\text { Body mass } \\
\text { index }\left(\mathrm{kg} / \mathrm{m}^{2}\right)\end{array}$ & $26.6 \pm 3.6$ & $25.7 \pm 2.9$ & 0.21 \\
\hline $\begin{array}{l}\text { Body mass index } \\
\geq 30 \mathrm{~kg} / \mathrm{m}^{2}(\%)\end{array}$ & 13.2 & 6.8 & 0.21 \\
\hline $\begin{array}{l}\text { Alaninoaminotransferase } \\
\text { (IU) }\end{array}$ & $88.1 \pm 73.8$ & $76.5 \pm 51.2$ & 0.30 \\
\hline Platelets $\left(10^{3} / \mathrm{mmc}\right)$ & $204.5 \pm 83.1$ & $212.2 \pm 65.5$ & 0.54 \\
\hline Cholesterol (mg/dl) & $174.0 \pm 34.0$ & $163.6 \pm 34.9$ & 0.04 \\
\hline Triglycerides (mg/dl) & $95.8 \pm 47.0$ & $77.9 \pm 31.6$ & 0.01 \\
\hline $\begin{array}{l}\text { Blood glucose } \\
(\mathrm{mg} / \mathrm{dl})\end{array}$ & $96.1 \pm 27.0$ & $93.7 \pm 19.3$ & 0.56 \\
\hline Insulin $(\mu \mathrm{U} / \mathrm{ml})$ & $13.8 \pm 9.4$ & $11.8 \pm 7.6$ & 0.30 \\
\hline HOMA & $3.39 \pm 2.25$ & $3.15 \pm 1.78$ & 0.49 \\
\hline Type 2 diabetes (\%) & 13.0 & 9.1 & 0.44 \\
\hline IL28B TT/TC/CC & 16.8/54.0/29.2 & $22.7 / 54.6 / 22.7$ & 0.48 \\
\hline PNPLA3 CC/CG/GG & $54.3 / 38.2 / 7.5$ & $59.1 / 29.5 / 11.4$ & 0.41 \\
\hline HCVRNA (LOG) & $5.9 \pm 0.6$ & $5.8 \pm 0.6$ & 0.45 \\
\hline \multicolumn{4}{|l|}{ Histology at biopsy } \\
\hline \multicolumn{4}{|l|}{ Steatosis grade (\%) } \\
\hline $\begin{array}{l}\text { Absent/mild- } \\
\text { moderate/severe }\end{array}$ & $54.8 / 28.8 / 16.4$ & $47.7 / 31.8 / 20.5$ & 0.63 \\
\hline \multicolumn{4}{|l|}{$\begin{array}{l}\text { Necroinflammatory } \\
\text { activity (\%) }\end{array}$} \\
\hline Severe & 43.8 & 38.6 & 0.50 \\
\hline \multicolumn{4}{|l|}{ Fibrosis (\%) } \\
\hline $\begin{array}{l}\text { Absent/mild- } \\
\text { moderate/severe }\end{array}$ & 30.9 & 25.0 & 0.40 \\
\hline
\end{tabular}

yrs, years; IU, international units; HOMA, homoeostasis model assessment. Data are given as mean \pm SD or as $\%$.

the aforementioned variants of IL28B (OR $0.69,95 \%$ CI $0.55-0.86, P=0.001$ ) and PNPLA3 (OR 1.84, 95\% CI $1.46-2.83, P<0.001$ ), but not of TM6SF2 (OR $1.48,95 \%$ CI $0.82-2.69, P=0.19$ ), were independently associated with the severity of steatosis (Table 2). Severity of steatosis was also associated with age (OR 1.02 , 95\% CI 1.01-1.03, $P<0.001$ ), BMI (OR 1.13, 95\% CI $1.08-1.18, P<0.001)$ and HOMA score (OR $1.08,95 \%$ CI $1.01-1.16, P=0.01)$. When T2DM was included in the model instead of HOMA, similar results were observed. The lack of association between the TM6SF2 variant and steatosis severity, as well as the independent link between the IL28B and PNPLA3 variants and steatosis severity, were further confirmed in subgroups of nondiabetic or nonobese patients (data not shown).

When we considered severe steatosis $(\geq 30 \%)$ as a dichotomous variable, BMI (OR 1.08, 95\% CI 1.02$1.14, P=0.007)$, HOMA score (OR $1.13,95 \%$ CI $1.02-1.25, P=0.01$ ) and the PNPLA3 variant (OR 1.98, $95 \%$ CI $1.38-2.84, P<0.001)$, but not the TM6SF2 variant (OR 1.49, 95\% CI $0.60-3.73, P=0.38$ ), were 


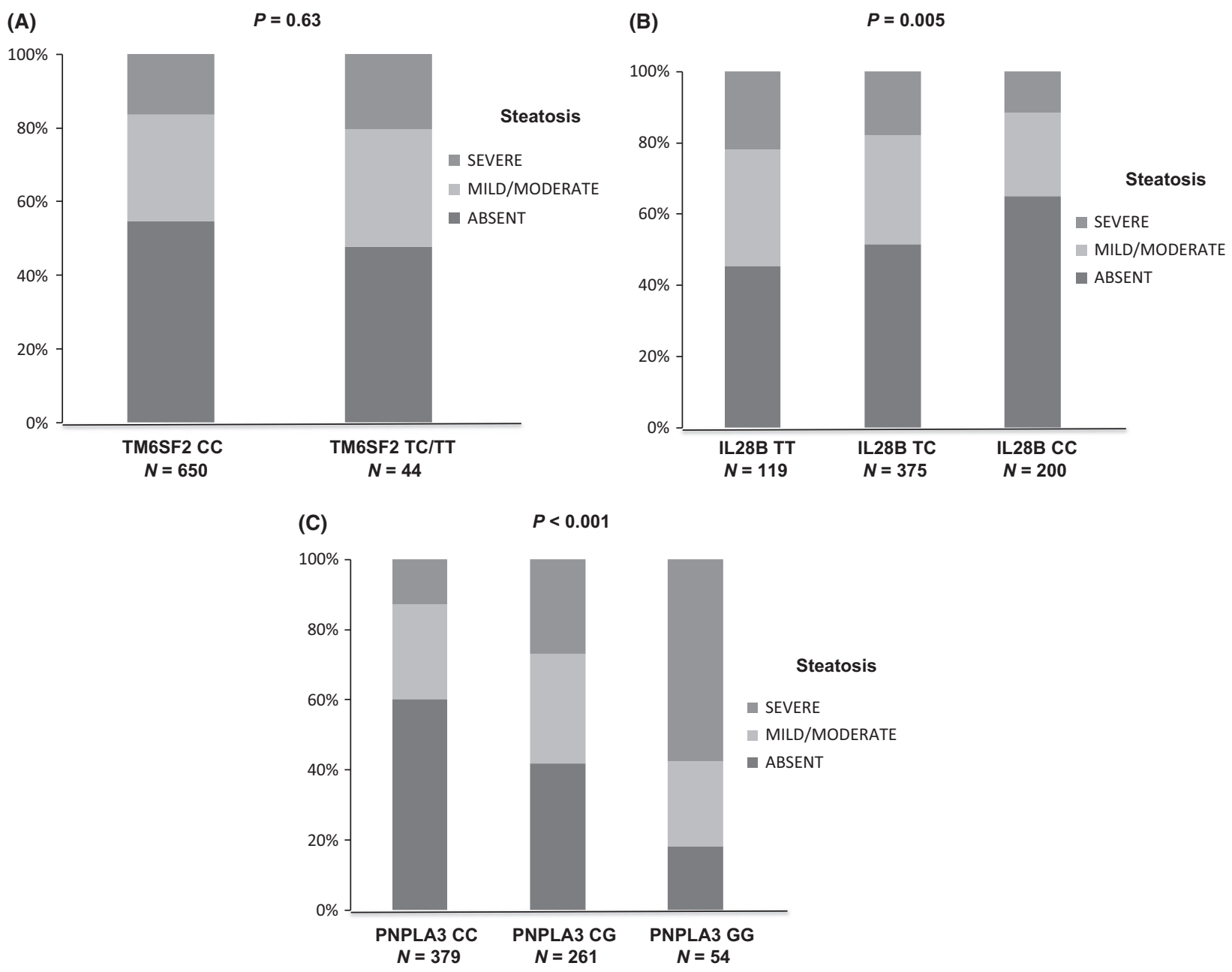

Fig. 1. Severity of steatosis according to TM6SF2 (A), IL28B (B) and PNPLA3 (C) genotypes.

Table 2. Factors associated with severity of steatosis in 694 patients with genotype 1 chronic hepatitis C

\begin{tabular}{|c|c|c|c|c|}
\hline \multirow[b]{2}{*}{ Variable } & \multicolumn{2}{|c|}{ Simple ordinal regression } & \multicolumn{2}{|c|}{ Multiple ordinal regression } \\
\hline & OR $(95 \% \mathrm{Cl})$ & $P$ value & OR $(95 \% \mathrm{Cl})$ & $P$ value \\
\hline Age (yrs) & $1.02(1.01-1.03)$ & $<0.001$ & $1.02(1.01-1.03)$ & $<0.001$ \\
\hline Female gender & $1.12(0.84-1.50)$ & 0.41 & $1.04(0.76-1.41)$ & 0.79 \\
\hline Body mass index $\left(\mathrm{kg} / \mathrm{m}^{2}\right)$ & $1.14(1.09-1.19)$ & $<0.001$ & $1.13(1.08-1.18)$ & $<0.001$ \\
\hline HOMA & $1.16(1.09-1.25)$ & $<0.001$ & $1.08(1.01-1.16)$ & 0.01 \\
\hline $\begin{array}{l}\text { TM6SF2 } \\
\text { CC/CT/TT }\end{array}$ & $1.32(0.74-2.34)$ & 0.34 & $1.48(0.82-2.69)$ & 0.19 \\
\hline $\begin{array}{l}\text { IL28B } \\
\text { TT/TC/CC }\end{array}$ & $0.66(0.53-0.82)$ & $<0.001$ & $0.69(0.55-0.86)$ & 0.001 \\
\hline $\begin{array}{l}\text { PNPLA3 } \\
\text { CC/CG/GG }\end{array}$ & $1.74(1.38-2.17)$ & $<0.001$ & $1.84(1.46-2.33)$ & $<0.001$ \\
\hline
\end{tabular}

yrs, years; IU, international units; HOMA, homoeostasis model assessment. Data are given as mean \pm SD or as \%.

independent risk factors by multivariate logistic regression analysis. Similarly, steatosis as continuous variable was independently associated with older age $(P=0.04)$, higher BMI $(P<0.001)$, higher HOMA score $(P=0.001)$, lack of the $I L 28 B$ C allele $(P=0.01)$ and presence of the PNPLA3 G allele $(P<0.001)$, but not with presence of the TM6SF2 T allele $(P=0.07)$, by multivariate linear regression analysis. 


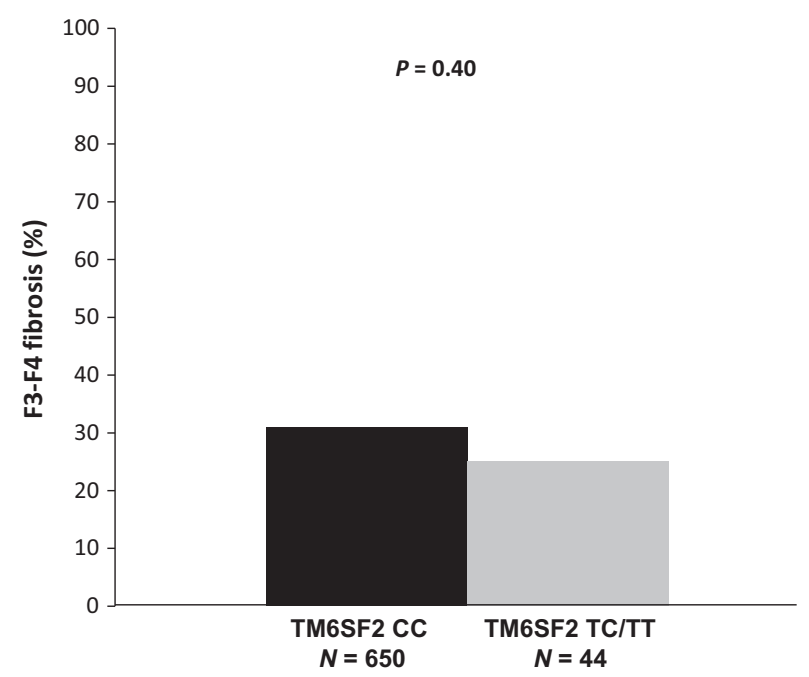

Fig. 2. Prevalence of F3-F4 fibrosis according to the TM6SF2 genotype.

Association of gene variants and steatosis severity with fibrosis severity in $\mathrm{CHC}$ patients

The prevalence of F3-F4 fibrosis was similar in patients with the TM6SF2 CC genotype compared to the CT genotype $(30.9 \%$ vs. $25 \%, P=0.40$; Fig. 2$)$. Simple logistic regression revealed that older age, higher HOMA score, severity of steatosis and severe necroinflammatory activity were associated with severe fibrosis $(P<0.01$, Table 3$)$. Multiple logistic regression confirmed that age (OR 1.04, 95\% CI 1.02-1.06, $P<0.001$ ), HOMA score (OR 1.23, 95\% CI 1.12-1.35, $P<0.001$ ), severity of steatosis (OR 1.36 , 95\% CI $1.06-1.75, P=0.01)$ and severe necroinflammatory activity (OR 3.55, 95\% CI 2.46-5.12, $P<0.001$ ), but not the TM6SF2 variant (OR 0.75, 95\% CI 0.34-1.63, $P=0.47$ ), the $I L 28 B$ variant (OR $0.98,95 \%$ CI
$0.74-1.29, P=0.90$ ), or the PNPLA3 variant (OR 0.99, 95\% CI $0.74-1.32, P=0.96)$, were independent predictors of severe fibrosis (Table 3 ). None of the gene variants remained associated with severe fibrosis when histological variables were removed from the model.

\section{Discussion}

We demonstrated that the TM6SF2 rs58542926 variant does not affect the severity of liver damage in terms of steatosis or fibrosis, using a large cohort of Caucasian patients with $\mathrm{G} 1 \mathrm{CHC}$ and a low prevalence of obesity. Furthermore, we confirmed the key roles of IL28B and PNPLA3 gene variants as steatosis modifiers.

Several lines of evidence in CHC have demonstrated that hepatic steatosis and some of its genetic determinants (e.g. PNPLA3 and IL28B gene variants) affect the severity of liver damage and the liver-related prognosis of patients $(3,15)$. Considering the recent evidence that TM6SF2 rs58542926 promotes steatosis and liver damage in NAFLD (17-20), we examined its potential role in $\mathrm{CHC}$. Our findings confirmed the low prevalence of the TM6SF2 variant in a population with G1 CHC, as well as the association of this gene variant with lower TC and TG levels $(17,19,20,26)$. We further confirmed the lack of an association between the TM6SF2 genotype and IR, as was reported in an elegant study by Zhou and colleagues (27). Unexpectedly, we found no association between the TM6SF2 variant and the presence or severity of steatosis in $\mathrm{G} 1 \mathrm{CHC}$ patients after adjusting for genetic and metabolic confounders. This result was further confirmed in subgroups of nondiabetic patients and nonobese patients, as well as by sorting steatosis at different thresholds.

The strength of our study lies in the large number of patients enrolled. To date, this study uses the largest cohort of G1 CHC patients to investigate the association between the TM6SF2 variant and steatosis in a clinical setting. Our results are not inconsistent with

Table 3. Factors associated with severe fibrosis in 694 patients with genotype 1 chronic hepatitis $C$

\begin{tabular}{|c|c|c|c|c|}
\hline \multirow[b]{2}{*}{ Variable } & \multicolumn{2}{|c|}{ Simple logistic regression } & \multicolumn{2}{|c|}{ Multiple logistic regression } \\
\hline & OR $(95 \% \mathrm{Cl})$ & $P$ value & OR $(95 \% \mathrm{Cl})$ & $P$ value \\
\hline Age (yrs) & $1.04(1.03-1.06)$ & $<0.001$ & $1.04(1.02-1.06)$ & $<0.001$ \\
\hline Female gender & $0.76(0.55-1.05)$ & 0.10 & $0.70(0.48-1.01)$ & 0.06 \\
\hline Body mass index $\left(\mathrm{kg} / \mathrm{m}^{2}\right)$ & $1.00(0.96-1.05)$ & 0.76 & $0.94(0.89-1.01)$ & 0.06 \\
\hline HOMA & $1.23(1.13-1.34)$ & $<0.001$ & $1.23(1.12-1.35)$ & $<0.001$ \\
\hline $\begin{array}{l}\text { TM6SF2 } \\
\text { CC/CT/TT }\end{array}$ & $0.74(0.36-1.50)$ & 0.41 & $0.75(0.34-1.63)$ & 0.47 \\
\hline $\begin{array}{l}\text { IL28B } \\
\text { TT/TC/CC }\end{array}$ & $0.94(0.74-1.20)$ & 0.64 & $0.98(0.74-1.29)$ & 0.90 \\
\hline $\begin{array}{l}\text { PNPLA3 } \\
\text { CC/CG/GG }\end{array}$ & $1.02(0.79-1.31)$ & 0.86 & $0.99(0.74-1.32)$ & 0.96 \\
\hline Severity of steatosis & $1.60(1.30-1.98)$ & $<0.001$ & $1.36(1.06-1.75)$ & 0.01 \\
\hline Severe necroinflammatory activity & $3.95(2.81-5.57)$ & $<0.001$ & $3.55(2.46-5.12)$ & $<0.001$ \\
\hline
\end{tabular}

yrs, years; IU, international units; HOMA, homoeostasis model assessment. Data are given as mean \pm SD or as \%. 
the exome-wide study by Kozlitina et al. (17). Although their study was population-based, we looked at the TM6SF2 gene variant as a risk factor for steatosis in a population of $\mathrm{CHC}$ patients, who have a higher incidence of steatosis compared to the general population (28).

On the other hand, our data do not agree with data from two recent studies on CHC $(21,22)$. Coppola and colleagues (21) found an association between steatosis and the TM6SF2 variant in a small cohort $(n=148)$ of $\mathrm{CHC}$ patients with different HCV genotypes. Similarly, Milano and colleagues (22) identified a link between the TM6SF2 variant and steatosis in $815 \mathrm{CHC}$ patients, confirming this association in the subgroup of $451 \mathrm{G} 1$ patients only. Several factors may account for the inconsistent results between studies, including the low number of patients evaluated in proportion to the low prevalence of the TM6SF2 variant (21), the inclusion of patients with different CHC genotypes $(21,22)$, as well as differences in histological methods $(21,22)$, mean BMI (22) and the percentage of patients with moderate alcohol intake (22). Our study results confirm the modulation of steatosis and its severity by variants in the PNPLA3 and IL28B genes in patients with CHC $(8,9,11,12)$.

The lack of an association between the TM6SF2 variant and steatosis in $\mathrm{CHC}$ may be explained by considering the mechanisms leading to steatosis in $\mathrm{CHC}$, and the mechanisms by which the TM6SF2 variant promotes liver fat accumulation. In patients with $\mathrm{CHC}$, steatosis is a prevalent histological finding related to metabolic and viral factors, including induction of IR (especially for G1 patients) and impairment of lipid export from the liver (especially for, but not exclusive to, G3 patients) (28). In experimental models, silencing TM6SF2 reduces the secretion of very low density lipoproteins and causes a predisposition to fatty liver development $(17,26)$. Therefore, although the TM6SF2 variant does not interfere with IR (27), both HCV infection and TM6SF2 promote steatosis by affecting lipid export from the liver, although by different pathways. Accordingly, we might hypothesize that the steatogenic effect of the TM6SF2 variant could be clinically less relevant in $\mathrm{CHC}$ patients.

We did not find any association between the TM6SF2 variant and liver fibrosis, as was observed in NAFLD patients (18-20) and CHC patients (22). In NAFLD, the link between fibrosis and the TM6SF2 variant is mediated by steatohepatitis. Thus, the lack of an effect of the TM6SF2 variant on liver fat accumulation in our CHC cohort could explain the absence of a link with the severity of fibrosis. In our population, PNPLA3 and $I L 28 B$ gene variants were not directly associated with F3-F4 fibrosis, although their effects were mediated by steatosis induction. Further studies are necessary to establish how the HCV genotype and other clinical characteristics impact the effect of the TM6SF2 variant on steatosis and liver damage in $\mathrm{CHC}$.
From a clinical perspective, our data confirm the roles of the PNPLA3 and IL28B gene variants, but not the TM6SF2 variant, as major disease-modifying factors in $\mathrm{CHC}$ that promote histological steatosis, which contributes to the progression of liver disease. If confirmed in other studies performed in other geographical and clinical settings, this relationship would suggest that genotyping of PNPLA3 and IL28B, but not TM6SF2, should be undertaken in $\mathrm{CHC}$ for risk stratification and personalized disease management, with more intensive follow-up of patients with the variant genotype, both before and after viral eradication.

The main limitation of this study lies in the potentially limited external validity of the results for different populations and settings. Thus, replicating our results is important. Lack of data on body fat distribution, serum levels of adipocytokines and other polymorphisms that could conceivably confound the data may have also affected the results. In conclusion, we found that in a large cohort of G1 CHC patients with a low prevalence of obesity, the TM6SF2 rs58542926 variant does not affect the severity of liver damage in terms of steatosis or fibrosis. However, we did confirm the key roles of IL28B and PNPLA3 gene variants as steatosis modifiers. These data, if further validated, could be useful in managing patients before and after therapy.

\section{Acknowledgements}

Financial support: None.

Conflict of interest: The authors do not have any disclosures to report.

\section{References}

1. Global Burden of Hepatitis C Working Group. Global burden of disease (GBD) for hepatitis C. J Clin Pharmacol 2004; 44: 20-9.

2. Petta S, Craxì A. Current and future HCV therapy: do we still need other anti-HCV drugs? Liver Int 2015; 35(Suppl. 1): $4-10$

3. Petta S, Amato M, Cabibi D, et al. Visceral adiposity index is associated with histological findings and high viral load in patients with chronic hepatitis $\mathrm{C}$ due to genotype 1 . Hepatology 2010; 52: 1543-52.

4. Everhart JE, Lok AS, Kim HY, et al. HALT-C Trial Group. Weight-related effects on disease progression in the hepatitis $\mathrm{C}$ antiviral long-term treatment against cirrhosis trial. Gastroenterology 2009; 137: 549-57.

5. Pekow JR, Bhan AK, Zheng H, Chung RT. Hepatic steatosis is associated with increased frequency of hepatocellular carcinoma in patients with hepatitis C-related cirrhosis. Cancer 2007; 109: 2490-6.

6. Romeo S, Kozlitina J, Xing C, et al. Genetic variation in PNPLA3 confers susceptibility to nonalcoholic fatty liver disease. Nat Genet 2008; 40: 1461-5.

7. Tanaka Y, Nishida N, Sugiyama M, et al. Genome-wide association of IL28B with response to pegylated interferon-alpha and ribavirin therapy for chronic hepatitis C. Nat Genet 2009; 41: 1105-9. 
8. Valenti L, Rumi M, Galmozzi E, et al. Patatin-like phospholipase domain-containing 3 I148M polymorphism, steatosis, and liver damage in chronic hepatitis C. Hepatology 2011; 53: 791-9.

9. Trépo E, Pradat P, Potthoff A, et al. Impact of patatin-like phospholipase-3 (rs738409 $\mathrm{C}>\mathrm{G}$ ) polymorphism on fibrosis progression and steatosis in chronic hepatitis C. Hepatology 2011; 54: 60-9.

10. Rüeger S, Bochud PY, Dufour JF, et al. Impact of common risk factors of fibrosis progression in chronic hepatitis C. Gut 2015; 64: 1605-15.

11. Cai T, Dufour JF, Muellhaupt B, et al. Swiss Hepatitis C CohortStudy Group. Viral genotype-specific role of PNPLA3, PPARG, MTTP, and IL28B in hepatitis C virusassociated steatosis. J Hepatol 2011; 55: 529-35.

12. Petta S, Rosso C, Leung R, et al. Effects of IL28B rs12979860 CC genotype on metabolic profile and sustained virologic response in patients with genotype 1 chronic hepatitis C. Clin Gastroenterol Hepatol 2013; 11: e1.

13. Chang KC, Tseng PL, Wu YY, et al. A Polymorphism in IFNL3 is an Independent Risk Factor for Development of Hepatocellular Carcinoma After Treatment of HCV Infection. Clin Gastroenterol Hepatol 2015; 13: 1017-24.

14. Sato M, Kondo M, Tateishi R, et al. Impact of IL28B genetic variation on HCV-induced liver fibrosis, inflammation, and steatosis: a meta-analysis. PLoS ONE 2014; 9: e91822.

15. Noureddin M, Wright EC, Alter HJ, et al. Association of IL28B genotype with fibrosis progression and clinical outcomes in patients with chronic hepatitis $\mathrm{C}$ : a longitudinal analysis. Hepatology 2013; 58: 1548-57.

16. Marabita F, Aghemo A, De Nicola S, et al. Genetic variation in the interleukin-28B gene is not associated with fibrosis progression in patients with chronic hepatitis $\mathrm{C}$ and known date of infection. Hepatology 2011; 54: 1127-34.

17. Kozlitina J, Smagris E, Stender S, et al. Exome-wide association study identifies a TM6SF2 variant that confers susceptibility to nonalcoholic fatty liver disease. Nat Genet 2014; 46: 352-6.

18. Sookoian S, Castaño GO, Scian R, et al. Genetic variation in transmembrane 6 superfamily member 2 and the risk of nonalcoholic fatty liver disease and histological disease severity. Hepatology 2015; 61: 515-25.

19. Dongiovanni P, Petta S, Maglio C, et al. TM6SF2 gene variant disentangles nonalcoholic steatohepatitis from cardiovascular disease. Hepatology 2015; 61: 506-14.

20. Liu YL, Reeves HL, Burt AD, et al. TM6SF2 rs58542926 influences hepatic fibrosis progression in patients with non-alcoholic fatty liver disease. Nat Commun 2014; 5: 4309.

21. Coppola N, Zampino R, Cirillo G, et al. Tm6sf2 e167k variant is associated with severe steatosis in chronic hepatitis c, regardless of pnapl3 polymorphism. Liver Int 2015; 35: 1959-63.

22. Milano M, Aghemo A, Mancina R, et al. TM6SF2 gene E167K variant impacts on steatosis and liver damage in chronic hepatitis C patients. Hepatology 2015; 62: 111-7.

23. American Diabetes Association. Standards of Medical Care in Diabetes-2014. Diabetes Care 2014; 37(Suppl. 1): S14-80.

24. Matthews DR, Hosker JP, Rudenski AS, et al. Homeostasis model assessment: insulin resistance and beta-cell function from fasting plasma glucose and insulin concentrations in man. Diabetologia 1985; 28: 412-9.

25. Scheuer PJ. Classification of chronic viral hepatitis: a need for reassessment. J Hepatol 1991; 13: 372-4.

26. Holmen OL, Zhang H, Fan Y, et al. Systematic evaluation of coding variation identifies a candidate causal variant in TM6SF2 influencing total cholesterol and myocardial infarction risk. Nat Genet 2014; 46: 345-51.

27. Zhou Y, Llauradó G, Oresic M, et al. Circulating triacylglycerol signatures insulin sensitivity in NAFLD associated with the E167K variant in TM6SF2. J Hepatol 2015; 62: 657-63.

28. Bugianesi E, Salamone F, Negro F. The interaction of metabolic factors with HCV infection: does it matter? J Hepatol 2012; 56(Suppl. 1): S56-65.

\section{Supporting information}

Additional Supporting Information may be found at onlinelibrary.wiley.com/doi/10.1111/liv.12918/suppinfo 